\title{
Implementation of ICP Slam Algorithm on Fire Bird V for Mapping of an Indoor Environment
}

\author{
Arpitha Shankar S I 1,*, M. Shivakumar², K.R Prakash ${ }^{3}$ \\ ${ }^{1}$ Dept. of Electronics and Communication Engineering, GSSSIETW, Mysuru, India \\ ${ }^{2}$ GSSSIETW, Mysuru, India \\ ${ }^{3}$ Dept. of Mechanical Engineering, NIE, Mysuru, India \\ *Corresponding author. Email: arpithasi@gsss.edu.in
}

\begin{abstract}
Mapping and Exploration are the fundamental tasks in many mobile robotic applications such as warehouse management, search and rescue operations in disaster scenarios, service robotics, patrolling and autonomous driving. Single robots are employed in the above-said tasks to model the environment accurately and perform complex autonomous navigation tasks. Due to robustness and fault-tolerant nature, multi-robot systems are preferred over single robots for exploration tasks. Each robot in the multi-robot system explores and builds the maps of the environment individual and merges the different robots' maps to build a global map. To create a map of an unknown environment, each robot should perform SLAM. Simultaneous Localization and Mapping (SLAM) is widely used in mobile robots for self-localization and mapping the environment. The ICP (Iterative Closest Point) is one of the best approaches for SLAM. The implementation of ICP-SLAM for multi-robot systems to map the indoor environment is described here. This method is tested on the Firebird V robot equipped with RPLiDAR.
\end{abstract}

Keywords: Iterative Closest Point, Mapping, Simultaneous Localization \& Mapping, and Self localization.

\section{INTRODUCTION}

Autonomous robots are just like humans, which can perform the task and make the decision. An autonomous robot perceives environment through sensors and makes decisions based on what it perceives and actuate the movement/manipulations within that environment. Autonomous multi-robot systems are preferred over single robots because they allow the parallel execution of tasks and are more efficient and roboust than single robots. Multi robot systems are employed in many dangerous environments such as search and rescue, chemical industries, oil plants etc. In these fields, robots should have the ability to navigate safely by avoiding hazardous situations and unsafe conditions (temperature, radiation, exposure to weather, etc.). When the robot wants to navigate to a particular place, they should know the representation of the surrounding scene, i.e. map of the environment and the ability to interpret that representation. From the map, a robot will come to know where the obstacle is and where the free space is; using that information robot will navigate to a particular destination safely. Simultaneous Localization and Mapping is a method that allows the robot to create a map of the surrounding environment and simultaneously localizing itself on the map.

Most of the SLAM technology uses LiDAR and camera sensor to map the environment. Camera is less preferred over LIDAR as it is sensitive to lightning effect, fog, snowfall and heavy rain, which minimizes the capability of the computer system to interpret the surrounding scene reliably. With camera, computational complexity involved in building the occupancy grid map is high, as it is susceptible to environmental lighting, and the camera field of view is limited. The LiDAR [1-2] plays a significant role in robot navigation because it can build occupancy grid maps with a low computational cost, as it does not require preprocessing techniques like smoothing, segmentation and filtering, uninfluenced by the illumination conditions and can be used in both indoor and outdoor environments.

Probabilistic[3] and Scan matching algorithms are the commonly used approach for SLAM problem. In this work, a scan matching algorithm is used to align the two scan data acquired using laser range finder when the robot is in motion. Scan alignment is essential in the context of mapping or SLAM. Mapping is incremental, 
and it is not possible to get the full view of the scene from single scan reading. As the robot navigates in the environment, it won't get a single sensor/LIDAR reading to observe the environment; it will get multiple scan data from different locations. If the robot does not know the exact position from where those scans are taken, it must apply transformation to build a consistent map (Shift and Rotate). Scan matching algorithms [4-6] are classified as

Point to point: Only points are used to obtain the correspondence. Correspondence means matching the closest point for each point in the source point cloud from reference point cloud.

Feature to feature: Features such as corners, segments etc. are used for obtaining the correspondence [7].

Point to feature: Points and features are used to obtain the correspondence [8].

Computationally expensive SLAM cannot be implemented on Firebird V due to the limited resources available on embedded hardware; hence, the most commonly used scan matching approach for SLAM is the ICP algorithm which Besl and McKay developed.

\section{RELATED WORKS}

Simultaneous Localization and Mapping (SLAM) is a research problem addressed by many authors over the decades. It arises when an autonomous moving vehicle estimates the map of the sourrounding environment and its relative pose. If the global position information is not known, then the pose estimatimation of the vehicle and mapping is inaccurate. Building of accurate map in realtime is a challenging task as the map contains many entities.

Smith and Cheeseman [9] used an extended Kalman filter (EKF) for solving SLAM. This paper describes a general method for estimating the nominal relationship assumed the model is linear with Gaussian distribution and expected error (covariance) between coordinate frames representing the relative locations of objects.

Newmann[10] in his paper proved that for EKF is suitable for linear SLAM problems, where the observation model and motion model are linear functions with Gaussian noise. Covariance matrices are quadratic in EKF; hence, updating them becomes complex while scaling SLAM to maps with more features.

M. Montemerlo et.al,[11] proposed an efficient FastSLAM algorithm that uses particle filters for estimating robot path. Each particle requires N lowdimensional EKFs for each of the $\mathrm{N}$ landmarks, which is different from the above SLAM algorithms, where single Gaussian is used to estimate locations of all features. FastSLAM algorithm requires $\mathrm{O}(\mathrm{NM})$ memory, where $\mathrm{M}$ is the number of particles in the particle filter. This filter requires $\mathrm{O}(\mathrm{M} \log \mathrm{N})$ time for updation. But in worst case, it is suspected that size of the map is exponential with number of prticles.

Apart from the probabilistic approach, a Scan matching technique plays a significant role in solving the SLAM problem. In scan matching, the robot's transformation between the two poses is determined by matching the scans taken from the sensors. Since the exploring sensors are usually very precise and fast, scan matching technique is very effective for a mobile robot to localize itself with respect to the given reference maps. Most widely used scan matching method is the Iterative Closest Point (ICP) method.

\section{ICP SLAM ALGORITHM}

In the ICP SLAM algorithm, the assumption is made to find the transformation between robot scans. The belief says that every point in the first scan corresponds to its closest point in the second scan. The solution is found using these correspondences.

Three important steps [12] associated with ICP algorithm are:

\section{Step 1: Laser Scan Projection}

To perform data association, it is necessary to represent all the scans in the same frame. In this step, every new scan carried out using laser is projected into reference frame using homogeneous transformation matrix.

Let $\mathrm{X}_{1}$ and $\mathrm{X}_{2}$ be two points in two different scans $\mathrm{SC}_{\text {ref }}$ and $\mathrm{SC}_{\text {new }}$ respectively. $\mathrm{SC}_{\text {ref }}$ is the scan which is taken initially, and $\mathrm{SC}_{\text {new }}$ took in while the robot is in motion. Using the following equations, $\mathrm{X}_{2}^{\prime}$ which is the projection of $\mathrm{X}_{2}$ in the global frame is obtained by:

$\mathrm{X}_{2}{ }^{1}=\mathrm{T} \times \mathrm{X}_{2}$
$T=\left[\begin{array}{ccc}\cos \theta & -\sin \theta & t x \\ \sin \theta & \cos \theta & t y \\ 0 & 0 & 1\end{array}\right]$

Where $\mathrm{T}=$ Homogeneous Transformation Matrix.

\section{Step 2: Data Association}

For each point in the new scan $\mathrm{SC}^{1}{ }_{\text {new }}$, its correspondent in the reference scan $\mathrm{SC}_{\text {ref }}$ is calculated here. That is accomplished by calculating the euclidean distances between one point of $\mathrm{SC}^{1}$ new and all the points of $\mathrm{SC}_{\text {ref. }}$ The point of $\mathrm{SC}_{\text {ref }}$ corresponding to the smallest distance is chosen to be the corresponding point.

\section{Pseudo code for data association}

$$
\begin{aligned}
& \text { for } i=1 \text { to } N \\
& \text { for } j=1 \text { to } N_{0} \\
& d x=X \text { 'snew(i) - Xsref (j) } \\
& d y=Y \text { 'snew(i) - Ysref (j) }
\end{aligned}
$$


if $\left(d_{\min }(\mathrm{i})>d(\mathrm{j})\right) \mathrm{d}_{\min }(\mathrm{i})=\mathrm{d}(\mathrm{j})$

end for

$\mathrm{r}(\mathrm{i})=\mathrm{j}$

end for

Where:

$-N$ : size of $\mathrm{SC}^{1}$ new.

$-N_{0}$ : size of $\mathrm{SC}_{\text {ref. }}$.

- X'snew: abscissas of $\mathrm{SC}^{1}{ }_{\text {new }}$ points.

- Xsref : abscissas of $\mathrm{SC}_{\text {ref }}$ points.

- Y'snew: ordinates of $\mathrm{SC}^{1}{ }_{\text {new }}$ points.

- Ysref : ordinates of $\mathrm{SC}_{\text {new }}$ points

$-\mathrm{d}_{\text {min }}$ : minimal distance between $\mathrm{SC}^{1}{ }_{\text {new }}$ points and $\mathrm{SC}_{\text {ref }}$ points.

$-\mathrm{r}$ (i): rank of a point of $\mathrm{SC}_{\text {ref }}$ which corresponds to the $i^{\text {th }}$ point in $\mathrm{SC}^{1}$ new.

\section{Step 3: Position estimation}

The criteria are formulated to minimize the mean square error of the distance between the correlated points[13-15]. The following equations are used for minimization.

$$
\begin{aligned}
& J=\frac{1}{N} \sum_{i=1}^{N}\left[\left(\left(X \operatorname{sref}(C(i))-X^{\prime} \text { snew }(i)\right)^{2}+\right.\right. \\
& \left.\left(Y \operatorname{sref}(C(i))-Y^{\prime} \text { snew }(i)\right)^{2}\right] \\
& X^{\prime} \operatorname{snew}(i)=X \operatorname{snew}(i) * \cos \theta-Y \operatorname{snew}(i) * \\
& \sin \theta+t_{x} \\
& Y^{\prime} \operatorname{snew}(i)=X \operatorname{snew}(i) * \sin \theta+Y \operatorname{snew}(i) * \\
& \cos \theta+t_{y}
\end{aligned}
$$

This step involves estimating the parameters of the transformation $\mathrm{T}\left(\mathrm{t}_{\mathrm{x}}, \mathrm{t}_{\mathrm{y}}\right.$ and $\left.\theta\right)$ by reducing the squared error of the distance between the two associated scans and rejecting the non-associated points by defining a binary function. Correct associations between the two points will be done using binary weight function, and the probability of having false is less during estimation.

The ICP algorithm uses the scan matching method to align two scans in an iterative manner $\left(\mathrm{SC}_{\mathrm{ref}}\right.$ and $\left.\mathrm{SC}_{\text {new }}\right)$. To carry out alignment between two scans, the ICP algorithm chooses the closest points as correspondences in each iteration and computes the homogeneous matrix of transformation $\mathrm{T}$ to carry out alignment. Figure 2 indicates the various stages of ICP algorithm.

The global map and associated parts of $\mathrm{SC}_{\text {new }}$ represent the same point in the environment. The nonassociated parts of $\mathrm{SC}_{\text {new }}$ are pushed onto the map, and it represents a new part of the environment that is not yet modelled.

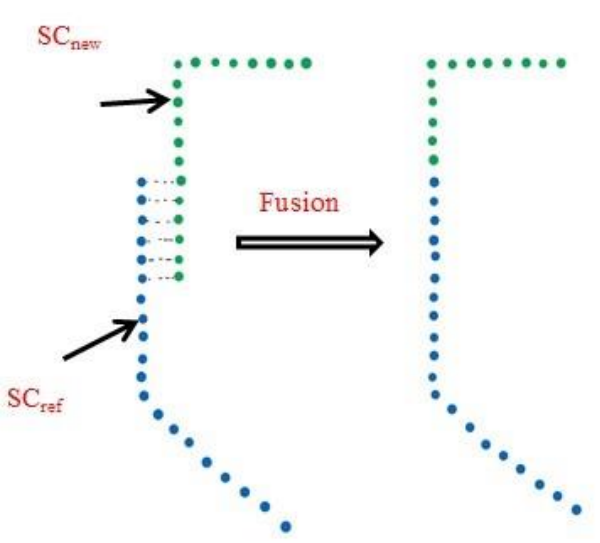

Figure 1 Data points fusion interpretation

\subsection{Flowchart of ICP algorithm}

Each robot will run the following steps independently to generate the map:

1. Initialization of robot position $\mathrm{P}(\mathrm{x}, \mathrm{y}, \mathrm{\Theta})$ to $(0,0,0)$ and update the GM(Global Map) by placing the first scan.

2. Robot will move to a new location $P_{\text {new }}$ and perform a new scan $\mathrm{SC}_{\text {new }}$ from the laser sensor.

3. ICP algorithm calculates the correlated location.

4. Last pose is used to translate $(x, y, \Theta)$ from source to reference frame.

5. Stich $\mathrm{SC}_{\text {new }}$ to map GM.

6. Repeat from step 2 until the robot stops.

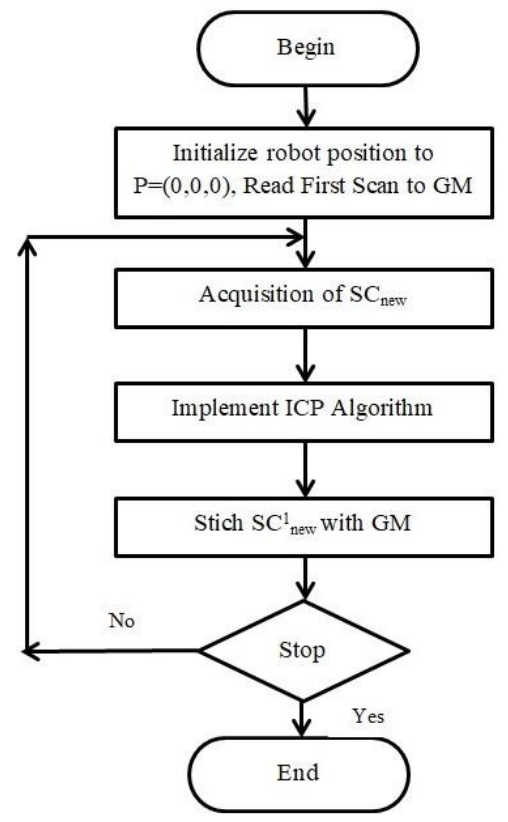

Figure 2 Flowchart of the ICP algorithm to generate the map 


\section{RESULTS AND DISCUSSION}

To generate map of the sourrounding environment, Firebird V ATMEGA2560 mobile robot equipped with LiDAR is used. Firebird V is a Universal Robotic Research Platform, which has been equipped with RPLIDAR A2 sensor which captures the information about the obstacles in the $18 \mathrm{~m}$ detection range. The scanning of the environment is done by driving the Firebird V fitted with LiDAR manually in the indoor environment using the rawlogger package of the MRPT. The scanned data from the LiDAR is as shown in the Figure 3.

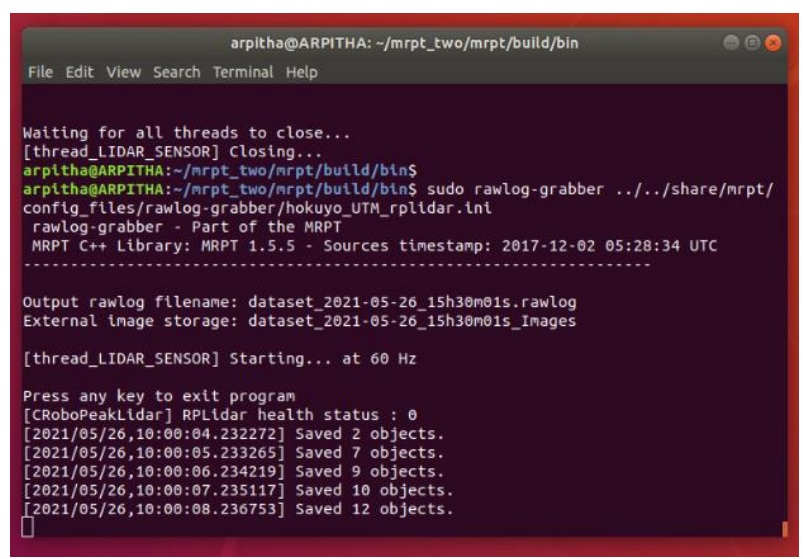

Figure 3 LiDAR performing scan in MRPT Platform

The Lidar sensor finds the distance to objects by rotating 360 , collects the scanned information in a point cloud format, which can be visualized in the MRPT tool as shown in Figure 4 and Figure 5. The visualization window shows point cloud data as well as the area it has explored. From Figure 4, it can be visualized that blue dots indicates obstacles such as walls and other objects. In contrast, free space shows no obstacles and unmapped regions.

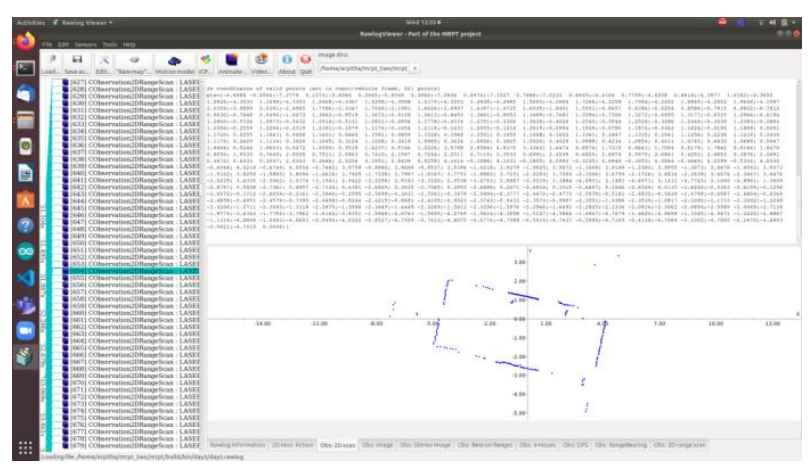

Figure 4 Scanned Data Visualization of room 1 in MRPT

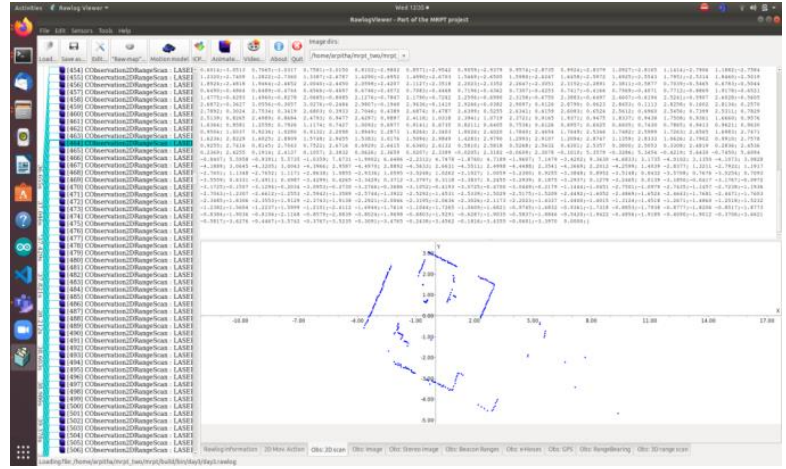

Figure 5 Scanned Data Visualization of room 2 in MRPT

The scanned information is fed to the ICP SLAM algorithm to generate the 2D map for an environment that the robot has explored. ICP SLAM algorithm applies a scan matching algorithm to align two iterative scans and locate itself in the map, as shown in Figure 6.

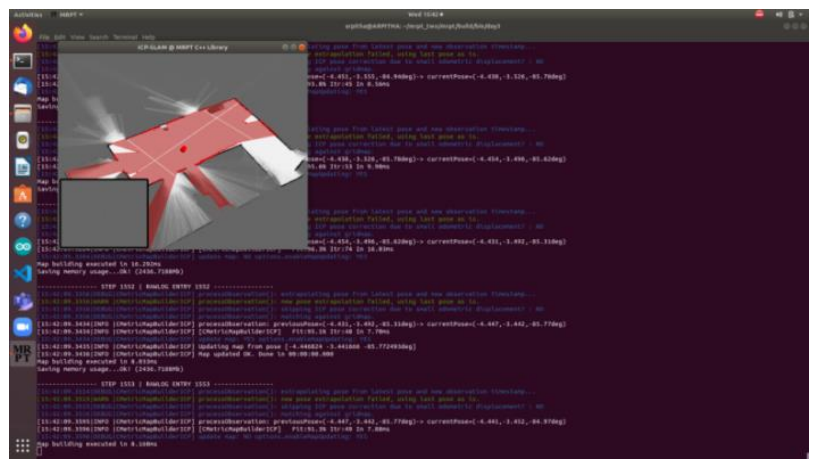

Figure 6(a)

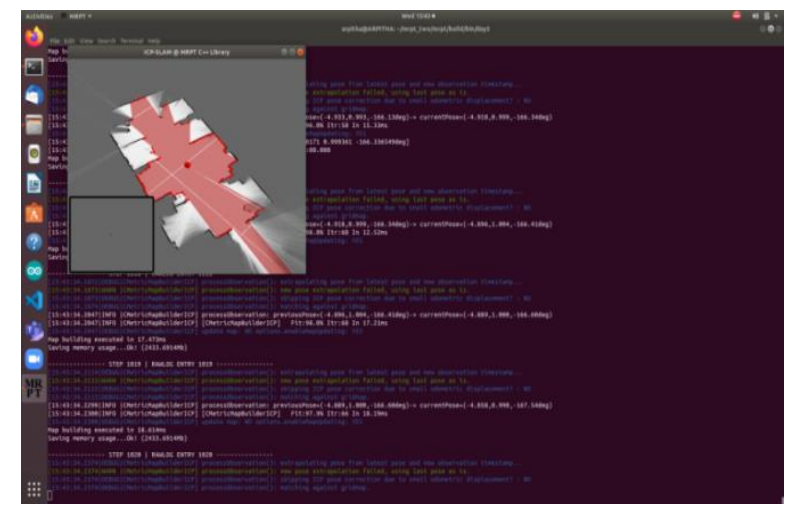

Figure 6(b)

Figure 6(a) \& 6(b) Map generation process using ICP SLAM in MRPT

The map in Figure 7 shows that the accurate map of the environment can be obtained by implementing simple ICP SLAM rather than applying probabilistic SLAM approaches as it involves more complexity in hardware and software. The robots use the generated maps for safe navigation purpose. 


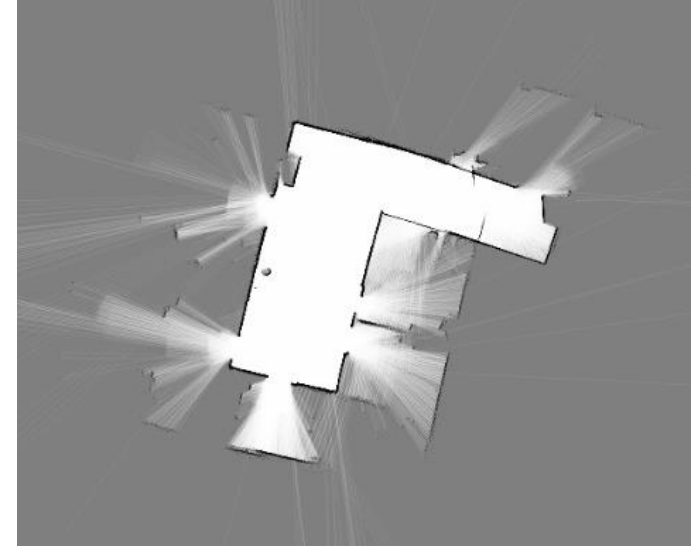

Figure 7(a)

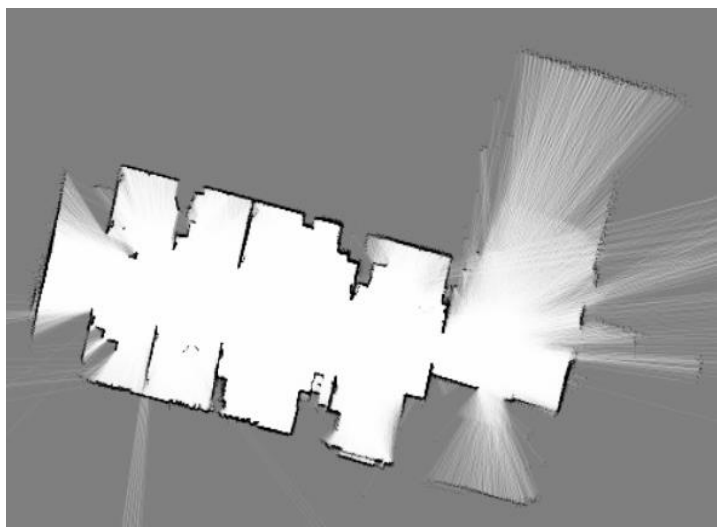

Figure 7(b)

Figure 7(a) \& (b) Generated Maps of the environment

\section{CONCLUSION}

This paper describes simultaneous localization and mapping for a multi-robot system where the environment is static. The aim is to provide an accurate map of the surrounding environments for safe navigation of the robot. We have implemented the ICP SLAM algorithm on two Firebird V robots equipped with LIDAR, which explores the surrounding environment independently and generates the map. Individual robots use these maps for navigation. In future works, map merging techniques are used to merge the map to create a global map for a multirobot scenario.

\section{REFERENCES}

[1] Chen, X, Milioto, A, Palazzolo, E.Giguere, P. Behley, J. Stachniss, C. SuMa++ Efficient LiDARbased Semantic SLAM. In Proceedings of the 2019 IEEE/RSJ International Conference on Intelligent Robots and Systems (IROS), Macau, China, 4-8 November 2019.

[2] Wang, Y.T.; Peng, C.C.; Ravankar, A.; Ravankar, A. A Single LiDAR-Based Feature Fusion Indoor Localization Algorithm. Sensors 2018, 18, 1294.
[3] Thrun S, Burgard W, and Fox D, "Probabilistic robotics," MIT press, 2005.

[4] S. Rusinkiewicz and M. Levoy, "Efficient variants of the ICP algorithm," International Conference on 3-D Digital Imaging and Modeling, 2001.

[5] Y.J. Lee, J.B. Song and J.H. Choi, "Performance improvement of iterative closest point-based outdoor SLAM by rotation invariant descriptors of salient regions," journal of intelligent and robotic systems, 2012.

[6] A. Segal, D. Haehnel, and S. Thrun, "GeneralizedICP," in Proceedings of Robotics: Science and Systems, Seattle, USA, 2009.

[7] K. Lingemann, H. Surmann, A. Nüchter, and J. Hertzberg, "Indoor and outdoor localization for fast mobile robots," in Proc. of IEEE/RSJ Internacional Conference on Intelligent Robots and Systems (IROS'04), vol. 2004, 2004, pp. 2185-2190.

[8] I. Cox, "Blanche-an experiment in guidance and navigation of an autonomous robot vehicle," Robotics and Automation, IEEE Transactionson, vol. 7, no. 2, pp. 193-204, 1991.

[9] Randall C. Smith and Peter Cheeseman. On the representation and estimation of spatial uncertainty. Int. J. Robotics Research,5(4), 1986.

[10] P. Newman. On the Structure and Solution of the Simultaneous Localisation and Map Building Problem. PhD thesis, Univ. of Sydney, 2000

[11] M. Montemerlo, S. Thrun, D. Koller, and B. Wegbreit. Fast- SLAM: A factored solution to the simultaneous localization and mapping problem. Proc. AAAI, 2002.

[12] F. Lu and E. Milios, "Robot pose estimation in unknown environments by matching $2 d$ range scans," Journal of Intelligent and Robotic Systems, vol.20, 1997, pp. 249-275.

[13] J. L. Martínez, J. González, J. Morales, A. Mandow, and A. J. García-Cerezo, "Mobile robot motion estimation by 2D scan matching with genetic and iterative closest point algorithms," Journal of Field Robotics, vol. 23, no. 1, pp. 21-34, 2006.

[14] L. Parker, G. Bekey, and J. Barhen, Eds , "Current state of the art in distributed autonomous mobile robots", in Distributed Autonomous Robotic Systems 4, New York: Springer, 2000, pp. 3-12.

[15] J. D. Tardos, J. Neira, P. M. Newman, and J. J. Leonard, "Robust mapping and localization in indoor environments using sonar data", Int. J. Robotics Research, (5(4)):311-330, 2002. 